\title{
Lie bialgebra quantizations of the oscillator algebra and their universal $R$-matrices
}

\author{
Angel Ballesteros and Francisco J. Herranz \\ Departamento de Física, Universidad de Burgos \\ Pza. Misael Bañuelos, \\ E-09001, Burgos, Spain
}

\begin{abstract}
All coboundary Lie bialgebras and their corresponding Poisson-Lie structures are constructed for the oscillator algebra generated by $\left\{N, A_{+}, A_{-}, M\right\}$. Quantum oscillator algebras are derived from these bialgebras by using the Lyakhovsky and Mudrov formalism and, for some cases, quantizations at both algebra and group levels are obtained, including their universal $R$-matrices.
\end{abstract}




\section{Introduction}

Deformed Heisenberg and oscillator algebras have recently focused many investigations coming from different directions. Among them, we would like to quote the construction of deformed statistics [1], the use of $q$-Heisenberg algebras to describe composite particles [2], the description of certain classes of exactly solvable potentials in terms of a $q$-Heisenberg dynamical symmetry [3], the link between deformed oscillator algebras and superintegrable systems [4, 5] and the relations between these deformed algebras and $q$-orthogonal polynomials [6].

Quantum universal enveloping algebras (QUEA) are much more selective deformations than general modifications of the commutation rules of a given algebra. In particular, the interest of finding Hopf algebra deformations of the oscillator algebra is twofold: firstly, because of the relevant role played by Hopf algebras to build up second quantization, as it has been recently discussed in [7]. On the other hand, a quasitriangular quantum oscillator algebra has been related to Yang-Baxter systems and link invariants in [8].

The aim of this paper is to provide a systematic study of the quantum universal enveloping oscillator algebras underlying possible further generalizations of these results. A brief summary of the oscillator algebra and group is given in section 2 . Since every QUEA defines uniquely a Lie bialgebra structure on the undeformed algebra, in section 3 we obtain and classify all coboundary Lie bialgebra structures for the harmonic oscillator algebra, as well as their corresponding Poisson-Lie brackets. In section 4 we make use of the Lyakhovsky and Mudrov formalism [9] in order to build up the deformed coproducts linked to all these Lie harmonic oscillator bialgebras. A complete quantization (including universal $R$-matrices) of two particular classes of non-standard (triangular) bialgebras is provided: the former is the natural "extension" of the non-standard deformation of the 1+1 Poincaré algebra discussed in [10 and the latter is a new three parameter quantization.

To our knowledge, the literature on Hopf algebra deformations of the oscillator algebra includes only the deformation given in [8, 11] and some new results that have been recently given in [12 by computing the dual of an arbitrary quantum oscillator group obtained by following an $R$-matrix approach in a particular matrix representation (see [13, 14, 15]). Among these known deformations, the former can be easily included within our clasification at the Lie bialgebra level, and can thus be obtained without making use of contraction procedures. On the other hand, our method gives explicit (and universal) expressions for the oscillator QUE algebras linked to the quantizations of [12] which are coboundaries. The procedure here

outlined precludes cumbersome duality computations and leads to rather simple candidates for universal $R$-matrices. 


\section{Classical oscillator algebra and group}

The oscillator Lie algebra $h_{4}$ is generated by $\left\{N, A_{+}, A_{-}, M\right\}$ with Lie brackets

$$
\left[N, A_{+}\right]=A_{+}, \quad\left[N, A_{-}\right]=-A_{-}, \quad\left[A_{-}, A_{+}\right]=M, \quad[M, \cdot]=0 .
$$

Besides the central generator $M$ there exists another Casimir invariant:

$$
C=2 N M-A_{+} A_{-}-A_{-} A_{+} .
$$

A $3 \times 3$ real matrix representation $D$ of (2.1) is given by:

$$
\begin{array}{ll}
D(N)=\left(\begin{array}{lll}
0 & 0 & 0 \\
0 & 1 & 0 \\
0 & 0 & 0
\end{array}\right), & D\left(A_{+}\right)=\left(\begin{array}{lll}
0 & 0 & 0 \\
0 & 0 & 1 \\
0 & 0 & 0
\end{array}\right), \\
D\left(A_{-}\right)=\left(\begin{array}{lll}
0 & 1 & 0 \\
0 & 0 & 0 \\
0 & 0 & 0
\end{array}\right), & D(M)=\left(\begin{array}{lll}
0 & 0 & 1 \\
0 & 0 & 0 \\
0 & 0 & 0
\end{array}\right) .
\end{array}
$$

The expression for a generic element of the oscillator group $H_{4}$ coming from this representation is:

$$
\begin{aligned}
T^{D} & =\exp \{m D(M)\} \exp \left\{a_{-} D\left(A_{-}\right)\right\} \exp \left\{a_{+} D\left(A_{+}\right)\right\} \exp \{n D(N)\} \\
& =\left(\begin{array}{ccc}
1 & a_{-} e^{n} & m+a_{-} a_{+} \\
0 & e^{n} & a_{+} \\
0 & 0 & 1
\end{array}\right)
\end{aligned}
$$

The group law for the coordinates $m, a_{-}, a_{+}$and $n$ is obtained by means of matrix multiplication $T^{D^{\prime \prime}}=T^{D^{\prime}} \cdot T^{D}$ :

$$
\begin{aligned}
& n^{\prime \prime}=n+n^{\prime}, \quad m^{\prime \prime}=m+m^{\prime}-a_{-} a_{+}^{\prime} e^{-n^{\prime}}, \\
& a_{+}^{\prime \prime}=a_{+}^{\prime}+a_{+} e^{n^{\prime}}, \quad a_{-}^{\prime \prime}=a_{-}^{\prime}+a_{-} e^{-n^{\prime}} .
\end{aligned}
$$

Left and right invariant vector fields are also deduced from (2.4) and read:

$$
\begin{gathered}
X_{N}^{L}=\partial_{n}, \quad X_{A_{+}}^{L}=e^{n} \partial_{a_{+}}, \quad X_{A_{-}}^{L}=e^{-n} \partial_{a_{-}}-a_{+} e^{-n} \partial_{m}, \quad X_{M}^{L}=\partial_{m} \\
X_{N}^{R}=\partial_{n}+a_{+} \partial_{a_{+}}+a_{-} \partial_{a_{-}}, \quad X_{A_{+}}^{R}=\partial_{a_{+}}-a_{-} \partial_{m}, \quad X_{A_{-}}^{R}=\partial_{a_{-}}, \quad X_{M}^{R}=\partial_{m} .
\end{gathered}
$$

The Heisenberg algebra can be seen as the subalgebra $\left\langle A_{+}, A_{-}, M\right\rangle$ of $h_{4}$ and the Heisenberg group $\left\langle a_{+}, a_{-}, m\right\rangle$ is recovered by taking the coordinate $n \equiv 0$ in $H_{4}$. Moreover, $h_{4}$ can be seen as a centrally extended $(1+1)$ Poincaré algebra (by $M$ ). This fact will be useful in the quantization process. 


\section{Coboundary oscillator Lie bialgebras}

Let $g$ be a Lie algebra and let $r$ be an element of $g \wedge g$. The cocomutator $\delta: g \rightarrow g \wedge g$ given by

$$
\delta(X)=[1 \otimes X+X \otimes 1, r], \quad X \in g,
$$

defines a coboundary Lie bialgebra $(g, \delta(r))$ if and only if $r$ fulfills the modified classical Yang-Baxter equation (YBE)

$$
[X \otimes 1 \otimes 1+1 \otimes X \otimes 1+1 \otimes 1 \otimes X,[[r, r]]]=0, \quad X \in g,
$$

where $[[r, r]]$ is the Schouten bracket defined by

$$
[[r, r]]:=\left[r_{12}, r_{13}\right]+\left[r_{12}, r_{23}\right]+\left[r_{13}, r_{23}\right],
$$

and, if $r=r^{i j} X_{i} \otimes X_{j}$, we have denoted $r_{12}=r^{i j} X_{i} \otimes X_{j} \otimes 1, r_{13}=r^{i j} X_{i} \otimes 1 \otimes X_{j}$ and $r_{23}=r^{i j} 1 \otimes X_{i} \otimes X_{j}$.

When the $r$-matrix is such that $[[r, r]]=0$ (classical YBE), we shall say that $(g, \delta(r))$ is a non-standard (or triangular) Lie bialgebra. On the contrary, a solution $r$ of (3.2) with non vanishing Schouten bracket will give rise to a so called standard Lie bialgebra.

We recall that, if $g=\operatorname{Lie}(G)$, the (unique) Poisson-Lie structure on $C^{\infty}(G)$ linked to a fixed bialgebra $(g, \delta(r))$ is given by the Sklyanin bracket

$$
\{\Psi, \Phi\}=r^{\alpha \beta}\left(X_{\alpha}^{L} \Psi X_{\beta}^{L} \Phi-X_{\alpha}^{R} \Psi X_{\beta}^{R} \Phi\right), \quad \Psi, \Phi \in C^{\infty}(G),
$$

where $X_{\alpha}^{L}$ and $X_{\tau}^{R}$ are the left and right invariant vector fields of $G$, respectively.

In particular, for $h_{4}$ we shall consider an arbitrary element $r$, which can be written in terms of six (real) coefficients:

$$
r=\alpha_{+} N \wedge A_{+}+\alpha_{-} N \wedge A_{-}+\vartheta N \wedge M+\xi A_{+} \wedge A_{-}+\beta_{+} A_{+} \wedge M+\beta_{-} A_{-} \wedge M
$$

It is a matter of computation to prove that the corresponding Schouten bracket for $r$ (3.5) is

$$
\begin{aligned}
{[[r, r]] } & =\alpha_{+}(\xi+\vartheta) N \wedge M \wedge A_{+}+\alpha_{-}(\xi-\vartheta) N \wedge M \wedge A_{-} \\
& -2 \alpha_{+} \alpha_{-} N \wedge A_{+} \wedge A_{-}+\left(\alpha_{+} \beta_{-}+\alpha_{-} \beta_{+}-\xi^{2}\right) M \wedge A_{+} \wedge A_{-}
\end{aligned}
$$

From this expression follows that the modified classical YBE (3.2) is fulfilled provided that:

$$
\begin{aligned}
& \alpha_{+} \alpha_{-}=0, \\
& \alpha_{+}(\xi+\vartheta)=0, \\
& \alpha_{-}(\xi-\vartheta)=0 .
\end{aligned}
$$

The solutions of this system are splitted into three classes: $\alpha_{+} \neq 0, \alpha_{-} \neq 0$ and $\alpha_{+}=\alpha_{-}=0$. For each of them we shall distinguish between non-standard $([[r, r]]=$ 0 ) and standard Lie bialgebras as follows. 
Type $\mathbf{I}_{+}$. If $\alpha_{+} \neq 0$ we have $\alpha_{-}=0$ and $\xi=-\vartheta$. The Schouten bracket reduces to:

$$
[[r, r]]=\left(\alpha_{+} \beta_{-}-\vartheta^{2}\right)\left(M \wedge A_{+} \wedge A_{-}\right) .
$$

Therefore if $\alpha_{+} \beta_{-}-\vartheta^{2} \neq 0$ we have standard solutions and when $\beta_{-}=\vartheta^{2} / \alpha_{+}$we are considering non-standard ones.

Type $\mathbf{I}_{-}$. If $\alpha_{-} \neq 0$ equations (3.7) imply $\alpha_{+}=0$ and $\xi=\vartheta$. The Schouten bracket is now:

$$
[[r, r]]=\left(\alpha_{-} \beta_{+}-\vartheta^{2}\right)\left(M \wedge A_{+} \wedge A_{-}\right) .
$$

Standard solutions are obtained when $\alpha_{-} \beta_{+}-\vartheta^{2} \neq 0$, while non-standard ones correspond to $\beta_{+}=\vartheta^{2} / \alpha_{-}$.

Type II. Finally, we consider the case with $\alpha_{+}=0$; if $\alpha_{-} \neq 0$ we are again in type $\mathrm{I}_{-}$, so we must take also $\alpha_{-}=0$ in order to have three disjoint sets of solutions. In this case equations (3.7) are automatically satisfied and the Schouten bracket is:

$$
[[r, r]]=-\xi^{2} M \wedge A_{+} \wedge A_{-}
$$

Then the condition $\xi \neq 0$ gives rise to standard solutions and $\xi=0$ to non-standard ones.

All the information concerning this classification of coboundary oscillator Lie bialgebras is summarized in table I. Poisson-Lie structures for the oscillator group are deduced via the Sklyanin bracket (3.4) and presented in table II.

Note that this classification is based in the use of skew-symmetric $r$-matrices. This implies no loss of generality: given an arbitrary element of $g \otimes g$, the map $\delta$ generated by (3.1) has to be skew-symmetric to give rise to a Lie bialgebra. This amounts to impose $A d^{\otimes 2}$-invariance on the symmetric part of $r$ and, therefore, $r$ will generate the same Lie bialgebra than its skew-symmetric part [16]. In particular, it can be easily checked that the more general element $\eta$ of $h_{4} \otimes h_{4}$ such that

$$
[X \otimes 1+1 \otimes X, \eta]=0, \quad X \in\left\{N, A_{+}, A_{-}, M\right\}
$$

is given by:

$$
\eta=\tau_{1}\left(N \otimes M+M \otimes N-A_{+} \otimes A_{-}-A_{-} \otimes A_{+}\right)+\tau_{2} M \otimes M,
$$

i.e., a linear combination of two terms directly related to the two Casimirs of $h_{4}$.

\section{Quantization}

In this section we first show how the Lyakhovsky and Mudrov (LM) formalism [9] allows all the cocommutators of the oscillator bialgebras previously found to generate coassociative coproducts in a straightforward way. Afterwards, we shall construct commutation rules and universal quantum $R$-matrices for some of these bialgebra quantizations. 


\subsection{The Lyakhovsky-Mudrov formalism}

Let us start with a short resume of the LM formalism which applies to an associative algebra $E$ over $\mathbf{C}$ with unit and generated by $n$ commuting elements $H_{i}$ and $m$ additional elements $X_{j}$. For any $m \times m$ numerical matrix $\mu$, by $\mu H$ we understand the matrix $\mu$ with all its entries multiplied by $H$. If $P$ is an $m \times m$ matrix with entries $p_{k l} \in E$, the $k$-th component of $P \dot{\otimes} \vec{X}$ is defined as

$$
(P \dot{\otimes} \vec{X})_{k}=\sum_{l=1}^{m} p_{k l} \otimes X_{l}
$$

The main LM statement [9] is that $E$ can be endowed with a coalgebra structure as follows (where we have denoted by $\sigma$ the permutation map $\sigma(a \otimes b)=b \otimes a$ ):

Proposition 1. Let $\left\{1, H_{1}, \ldots, H_{n}, X_{1}, \ldots, X_{m}\right\}$ a basis of an associative algebra $E$ over $\mathbf{C}$ verifying the conditions

$$
\left[H_{i}, H_{j}\right]=0, \quad i, j=1, \ldots, n .
$$

Let $\mu_{i}, \nu_{j}(i, j=1, \ldots, n)$ be a set of $m \times m$ complex matrices such that

$$
\left[\mu_{i}, \nu_{j}\right]=\left[\mu_{i}, \mu_{j}\right]=\left[\nu_{i}, \nu_{j}\right]=0, \quad i, j=1, \ldots, n .
$$

Let $\vec{X}$ be a column vector with components $X_{l}(l=1, \ldots, m)$. The coproduct and the counit

$$
\begin{aligned}
& \Delta(1)=1 \otimes 1, \quad \Delta\left(H_{i}\right)=1 \otimes H_{i}+H_{i} \otimes 1, \\
& \Delta(\vec{X})=\exp \left(\sum_{i=1}^{n} \mu_{i} H_{i}\right) \dot{\otimes} \vec{X}+\sigma\left(\exp \left(\sum_{i=1}^{n} \nu_{i} H_{i}\right) \dot{\otimes} \vec{X}\right) \\
& \epsilon(1)=1, \quad \epsilon\left(H_{i}\right)=\epsilon\left(X_{l}\right)=0, \quad i=1, \ldots, n ; \quad l=1, \ldots m ;
\end{aligned}
$$

endow $(E, \Delta, \epsilon)$ with a coalgebra structure.

The resulting coalgebra can be seen as a multiparametric deformation where the deformation parameters are the entries of the matrices $\mu_{i}$ and $\nu_{j}$. If we are able to find a compatible multiplication with the coproduct (4.4) we will have finally obtained a quantum algebra.

It is worth remarking that this formalism encodes in the set of matrices $\mu_{i}$ and $\nu_{j}$ the whole coalgebra structure. In fact, the role of these matrices is, essentially, to reflect the Lie bialgebra underlying a given quantum deformation. This can be clearly appreciated by taking the first order (in all the parameters) of (4.4):

$$
\Delta_{(1)}(\vec{X})=\left(\sum_{i=1}^{n} \mu_{i} H_{i}\right) \dot{\otimes} \vec{X}+\sigma\left(\left(\sum_{i=1}^{n} \nu_{i} H_{i}\right) \dot{\otimes} \vec{X}\right)
$$

and recalling that the cocommutator $\delta$ corresponds to the co-antisymmetric part of (4.6). It can be written in "matrix" form as:

$$
\delta(\vec{X})=\Delta_{(1)}(\vec{X})-\sigma \circ \Delta_{(1)}(\vec{X}) .
$$


We would like to emphasize the following points:

- The commuting elements $H_{i}$ are the primitive generators.

- The cocommutator $\delta\left(X_{i}\right)$ does not contain terms of the form $H_{i} \wedge H_{j}$.

- The same cocommutator (4.7) can be obtained from different choices of the matrices $\mu_{i}$ and $\nu_{j}$. This means that different sets of matrices might lead to right quantizations, all of them having the same first order terms in the deformation parameters. Moreover, we can choose $\mu_{i}=0$ as a representative of all these quantizations and we shall obtain

$$
\left.\delta(\vec{X})=-\left(\sum_{i=1}^{n} \nu_{i} H_{i}\right) \dot{\wedge} \vec{X}=-\left(\sum_{i=1}^{n} \nu_{i} H_{i}\right) \dot{\otimes} \vec{X}+\sigma\left(\sum_{i=1}^{n} \nu_{i} H_{i}\right) \dot{\otimes} \vec{X}\right) .
$$

Now let us reverse somehow the LM formalism trying to find in which way the oscillator Lie bialgebras given in table I can be recovered by a suitable choice of the matrices $\mu_{i}$ and $\nu_{j}$. Of course, the benefit of such a situation is to be able to "exponentiate" directly the bialgebra (4.8) to a full coalgebra (4.4 4.5).

Let us start with non-standard type $\mathrm{I}_{+}$oscillator bialgebras. By denoting $H_{1} \equiv$ $A_{+}, H_{2} \equiv M, X_{1} \equiv N, X_{2} \equiv A_{-}$, we see that $\left[H_{1}, H_{2}\right]=0$ and there exists a term of the type $H_{1} \wedge H_{2} \equiv A_{+} \wedge M$ within the cocommutator $\delta(N)$; however, this obstruction can be circumvented by defining a new generator in the form:

$$
N^{\prime}=N-\left(\beta_{+} / \alpha_{+}\right) M .
$$

Hence, the cocommutators for the non-primitive generators $N^{\prime}$ and $A_{-}$can be written as

$\delta\left(\begin{array}{c}N^{\prime} \\ A_{-}\end{array}\right)=\left(\begin{array}{cc}-\alpha_{+} A_{+} & 0 \\ 0 & -\alpha_{+} A_{+}\end{array}\right) \dot{\wedge}\left(\begin{array}{c}N^{\prime} \\ A_{-}\end{array}\right)+\left(\begin{array}{cc}0 & \left(\vartheta^{2} / \alpha_{+}\right) M \\ -\alpha_{+} M & -2 \vartheta M\end{array}\right) \dot{\wedge}\left(\begin{array}{c}N^{\prime} \\ A_{-}\end{array}\right)$

In view of this expression, the matrices $\mu_{i}$ and $\nu_{j}$ can be chosen as:

$$
\mu_{1}=\mu_{2}=\left(\begin{array}{ll}
0 & 0 \\
0 & 0
\end{array}\right), \quad \nu_{1}=\left(\begin{array}{cc}
\alpha_{+} & 0 \\
0 & \alpha_{+}
\end{array}\right), \quad \nu_{2}=\left(\begin{array}{cc}
0 & -\vartheta^{2} / \alpha_{+} \\
\alpha_{+} & 2 \vartheta
\end{array}\right) .
$$

Now, the set of conditions of Proposition 1 are fulfilled, and we can use this result to get the coproducts:

$$
\begin{gathered}
\Delta\left(\begin{array}{c}
N^{\prime} \\
A_{-}
\end{array}\right)=\exp \left\{\left(\begin{array}{ll}
0 & 0 \\
0 & 0
\end{array}\right)\right\} \dot{\otimes}\left(\begin{array}{c}
N^{\prime} \\
A_{-}
\end{array}\right) \\
+\sigma\left(\exp \left\{\left(\begin{array}{cc}
\alpha_{+} A_{+} & -\left(\vartheta^{2} / \alpha_{+}\right) M \\
\alpha_{+} M & \alpha_{+} A_{+}+2 \vartheta M
\end{array}\right)\right\} \dot{\otimes}\left(\begin{array}{c}
N^{\prime} \\
A_{-}
\end{array}\right)\right) \\
=\left(\begin{array}{c}
1 \otimes N^{\prime}+N^{\prime} \otimes(1-\vartheta M) e^{\alpha_{+} A_{+}+\vartheta M}-\left(\vartheta^{2} / \alpha_{+}\right) A_{-} \otimes M e^{\alpha_{+} A_{+}+\vartheta M} \\
1 \otimes A_{-}+A_{-} \otimes(1+\vartheta M) e^{\alpha_{+} A_{+}+\vartheta M}+\alpha_{+} N^{\prime} \otimes M e^{\alpha_{+} A_{+}+\vartheta M}
\end{array}\right)
\end{gathered}
$$

We can finally return to the initial basis elements, thus obtaining a three-parameter QUEA (denoted by $U_{\alpha_{+}, \vartheta, \beta_{+}}^{\left(I_{+} n\right)}\left(h_{4}\right)$ ) such that:

$$
\Delta(N)=1 \otimes N+N \otimes(1-\vartheta M) e^{\alpha_{+} A_{+}+\vartheta M}-\left(\vartheta^{2} / \alpha_{+}\right) A_{-} \otimes M e^{\alpha_{+} A_{+}+\vartheta M}
$$




$$
\begin{aligned}
& +\left(\beta_{+} / \alpha_{+}\right) M \otimes\left(1-(1-\vartheta M) e^{\alpha_{+} A_{+}+\vartheta M}\right) \\
\Delta\left(A_{-}\right)=1 \otimes & A_{-}+A_{-} \otimes(1+\vartheta M) e^{\alpha_{+} A_{+}+\vartheta M} \\
& +\left(\alpha_{+} N-\beta_{+} M\right) \otimes M e^{\alpha_{+} A_{+}+\vartheta M}
\end{aligned}
$$

This quantization procedure can be applied to the remaining types of bialgebras in the same way. For the standard type $I_{+}$bialgebras we also use (4.9), while for the bialgebras of type $I_{-}$we introduce the new generator

$$
N^{\prime}=N-\left(\beta_{-} / \alpha_{-}\right) M
$$

On the contrary, no such a kind of transformation is necessary to get the coproducts for the Lie bialgebras of type II.

The coproducts for the corresponding QUEA of the coboundary oscillator Lie bialgebras of table I are written down in table III; we denote each multiparametric quantum coalgebra by $U_{\alpha_{i}}^{(t m)}\left(h_{4}\right)$ where $t$ is the type, $m=s$ or $m=n$ according either to the standard or non-standard oscillator deformations and with $\alpha_{i}$ being the deformation parameters. The explicit expressions for the coproducts of $U_{\alpha_{+}, \vartheta, \beta_{+}, \beta_{-}}^{\left(I_{+} s\right)}\left(h_{4}\right)$ and $U_{\alpha_{-}, \vartheta, \beta_{+}, \beta_{-}}^{\left(I_{-}\right)}\left(h_{4}\right)$ are rather complicated so we keep their matrix forms written in terms of the generator $N^{\prime}$ defined by either (4.9) or by (4.14), respectively.

The final step in the quantization process of a fixed bialgebra is to find the commutation relations compatible with its deformed coproduct (counit and antipode can be obtained in the form explained in 91). In the following, we solve completely this problem and construct the deformed Hopf algebras $U_{\alpha_{i}}^{(t m)}\left(h_{4}\right)$ for some representative cases among the ones included in Table III.

\subsection{Non-standard type $\mathbf{I}_{+}: U_{z}^{(n)}\left(h_{4}\right)$}

It is remarkable that the oscillator algebra with basis $\left\{N, A_{+}, A_{-}, M\right\}$ can be interpreted as an extended $(1+1)$ Poincaré algebra where $N$ is the boost generator, $A_{+}$ and $A_{-}$generate the translations along the light-cone and $M$ is the central generator. This fact rises the question about whether it is possible to implement in this extended case the universal (non-standard) quantum deformation of the Poincaré algebra studied in [10] from a $T$-matrix approach.

Let us consider the non-standard oscillator bialgebras of type $\mathrm{I}_{+}$with $\vartheta=\beta_{+}=0$ and $\alpha_{+} \equiv z$. According to table I the Lie bialgebra is characterized by commutation relations (2.1), classical $r$-matrix:

$$
r=z N \wedge A_{+}
$$

and cocommutators:

$$
\begin{aligned}
& \delta\left(A_{+}\right)=0, \quad \delta(M)=0, \quad \delta(N)=z N \wedge A_{+} \\
& \delta\left(A_{-}\right)=z\left(A_{-} \wedge A_{+}+N \wedge M\right) .
\end{aligned}
$$


Poisson-Lie brackets are easily deduced from table II:

$$
\begin{aligned}
& \left\{n, a_{+}\right\}=z\left(e^{n}-1\right), \quad\left\{n, a_{-}\right\}=0, \quad\left\{a_{-}, a_{+}\right\}=z a_{-}, \\
& \{n, m\}=z a_{-}, \quad\left\{a_{+}, m\right\}=z a_{-} a_{+}, \quad\left\{a_{-}, m\right\}=-z a_{-}^{2} .
\end{aligned}
$$

A quantum deformation for this Lie bialgebra is given by the following statement:

Proposition 2. The coproduct $\Delta$, counit $\epsilon$, antipode $\gamma$

$$
\begin{gathered}
\Delta\left(A_{+}\right)=1 \otimes A_{+}+A_{+} \otimes 1, \quad \Delta(M)=1 \otimes M+M \otimes 1 \\
\Delta(N)=1 \otimes N+N \otimes e^{z A_{+}}, \quad \Delta\left(A_{-}\right)=1 \otimes A_{-}+A_{-} \otimes e^{z A_{+}}+z N \otimes M e^{z A_{+}} \\
\epsilon(X)=0, \\
X \in\left\{N, A_{+}, A_{-}, M\right\} \\
\gamma\left(A_{+}\right)=-A_{+}, \quad \gamma(M)=-M, \\
\gamma(N)=-N e^{-z A_{+}}, \quad \gamma\left(A_{-}\right)=-A_{-} e^{-z A_{+}}+z N M e^{-z A_{+}}
\end{gathered}
$$

and the commutation relations

$$
\left[N, A_{+}\right]=\frac{e^{z A_{+}}-1}{z}, \quad\left[N, A_{-}\right]=-A_{-}, \quad\left[A_{-}, A_{+}\right]=M e^{z A_{+}}, \quad[M, \cdot]=0
$$

determine a Hopf algebra (denoted by $U_{z}^{(n)}\left(h_{4}\right)$ ) which quantizes the non-standard bialgebra generated by the classical r-matrix (4.15).

The coproduct (4.18) is obtained from table III. Note that $M$ remains as a central generator. There is another element belonging to the center of $U_{z}^{(n)} h_{4}$ whose classical limit is (2.2), namely

$$
C_{z}=2 N M+\frac{e^{-z A_{+}}-1}{z} A_{-}+A_{-} \frac{e^{-z A_{+}}-1}{z} .
$$

An important feature of the quantum algebra $U_{z}^{(n)}\left(h_{4}\right)$ is that the generators $N$ and $A_{+}$form a Hopf subalgebra which coincides exactly with the corresponding to the quantum Poincaré algebra of [10]. We recall that for this Hopf subalgebra there is a universal $R$-matrix given by:

$$
R=\exp \left\{-z A_{+} \otimes N\right\} \exp \left\{z N \otimes A_{+}\right\}
$$

Obviously, (4.23) satisfies the quantum YBE for $U_{z}^{(n)} h_{4}$, but moreover it verifies

$$
\sigma \circ \Delta(X)=R \Delta(X) R^{-1}, \quad \text { for } \quad X \in\left\{N, A_{+}, A_{-}, M\right\} .
$$

This assertion must be proved only for $M$ and $A_{-}$; the proof for the former is trivial since it is a central generator, and for the latter we have

$$
\begin{aligned}
& \exp \left\{z N \otimes A_{+}\right\} \Delta\left(A_{-}\right) \exp \left\{-z N \otimes A_{+}\right\}=1 \otimes A_{-}+A_{-} \otimes 1=\Delta_{0}\left(A_{-}\right) \\
& \exp \left\{-z A_{+} \otimes N\right\} \Delta_{0}\left(A_{-}\right) \exp \left\{z A_{+} \otimes N\right\}=\sigma \circ \Delta\left(A_{-}\right)
\end{aligned}
$$


The fulfillment of relation (4.24) allows to use the FRT approach in order to get a quantum deformation of $F u n\left(H_{4}\right)$ by taking into account that in the matrix representation (2.3) the universal $R$-matrix (4.23) collapses into:

$$
D(R)=I \otimes I+z\left(D(N) \otimes D\left(A_{+}\right)-D\left(A_{+}\right) \otimes D(N)\right),
$$

where $I$ is the $3 \times 3$ identity matrix. Therefore, the Hopf structure of the associated oscillator quantum group is given by:

Proposition 3. The coproduct, counit, antipode

$$
\begin{gathered}
\Delta(\hat{n})=1 \otimes \hat{n}+\hat{n} \otimes 1, \\
\Delta\left(\hat{a}_{+}\right)=e^{\hat{n}} \otimes \hat{a}_{+}+\hat{a}_{+} \otimes 1, \\
\Delta\left(\hat{a}_{-}\right)=e^{-\hat{n}} \otimes \hat{a}_{-}+\hat{a}_{-} \otimes 1, \\
\Delta(\hat{m})=1 \otimes \hat{m}+\hat{m} \otimes 1-e^{-\hat{n}} \hat{a}_{+} \otimes \hat{a}_{-} ; \\
\epsilon(X)=0, \quad X \in\left\{\hat{n}, \hat{a}_{+}, \hat{a}_{-}, \hat{m}\right\} ; \\
\gamma(\hat{n})=-\hat{n}, \quad \gamma\left(\hat{a}_{+}\right)=-e^{-\hat{n}} \hat{a}_{+}, \\
\gamma\left(\hat{a}_{-}\right)=-e^{\hat{n}^{n}} \hat{a}_{-}, \quad \gamma(\hat{m})=-\hat{m}-\left(e^{-\hat{n}} \hat{a}_{+} e^{\hat{n}}\right) \hat{a}_{-} ;
\end{gathered}
$$

together with the commutation relations

$$
\begin{aligned}
& {\left[\hat{n}, \hat{a}_{+}\right]=z\left(e^{\hat{n}}-1\right), \quad\left[\hat{n}, \hat{a}_{-}\right]=0, \quad\left[\hat{a}_{-}, \hat{a}_{+}\right]=z \hat{a}_{-},} \\
& {[\hat{n}, \hat{m}]=z \hat{a}_{-}, \quad\left[\hat{a}_{+}, \hat{m}\right]=z \hat{a}_{-} \hat{a}_{+}, \quad\left[\hat{a}_{-}, \hat{m}\right]=-z \hat{a}_{-}^{2},}
\end{aligned}
$$

constitute a Hopf algebra denoted by $\operatorname{Fun}_{z}^{(n)}\left(H_{4}\right)$.

The coproduct (4.27), counit (4.28) and antipode (4.29) are obtained from the relations $\Delta(T)=T \dot{\otimes} T, \epsilon(T)=I$ and $\gamma(T)=T^{-1}$, where $T \equiv T^{D}$ is the generic element of the oscillator group $H_{4}$ (2.4). The commutation rules are deduced from $R T_{1} T_{2}=T_{2} T_{1} R$, where $T_{1}=T \otimes I, T_{2}=I \otimes T$ and $R$ given by (4.26).

The commutation relations $(4.30)$ can be seen as a Weyl quantization $\{,\} \rightarrow$ $z^{-1}[$,$] of the fundamental Poisson brackets (4.17). It is also clear that the coalgebra$ structure of $\operatorname{Fun}_{z}^{(n)}\left(H_{4}\right)$ determined by the coproduct (4.27) and counit (4.28) is valid for any quantum group which deforms $F u n\left(H_{4}\right)$.

Some features of this new quantum oscillator algebra can be emphasized:

- When the central extension $M$ and its corresponding quantum coordinate $\hat{m}$ vanish all results concerning the quantum Poincaré algebra and group given in [10 are recovered. In this sense, the quantum coordinates $\hat{n}, \hat{a}_{+}$and $\hat{a}_{-}$close a quantum Hopf subalgebra which coincides exactly with the quantum Poincaré group just mentioned.

- The primitive generator involved in the deformation is now $A_{+}$. This fact will be relevant at a representation theory level and, consequently, from the point of view of the physical properties of this deformed oscillator.

- The deformed Heisenberg subalgebra generated by $A_{+}, A_{-}$and $M$ is not a Hopf subalgebra due to the appearence of $N$ in $\Delta\left(A_{-}\right)$. However, the Hopf subalgebra 
structure can be recovered by working on a representation where the central generator $M$ is expressed as a multiple of the identity. In this situation, $N$ can be defined in terms of $A_{+}$and $A_{-}$by using the Casimir (4.22). In general, this type of non-standard deformed bosons can be expected to build up $q$-boson realizations of the already known non-standard quantum algebras [17, 18].

\subsection{Non-standard type II: $U_{\vartheta, \beta_{+}, \beta_{-}}^{(I I n)}\left(h_{4}\right)$}

The classical $r$-matrix

$$
r=\vartheta N \wedge M+\beta_{+} A_{+} \wedge M+\beta_{-} A_{-} \wedge M
$$

originates a non-standard three-parametric oscillator bialgebra of type II whose cocommutators and associated Poisson-Lie brackets appear in tables I and II, respectively. A quantum deformation of this coboundary Lie bialgebra is given by:

Proposition 4. The Hopf algebra denoted by $U_{\vartheta, \beta_{+}, \beta_{-}}^{(I I n)}\left(h_{4}\right)$ which quantizes the oscillator bialgebra generated by (4.31) has coproduct given in table III, counit (4.19), antipode

$$
\begin{aligned}
& \gamma(M)=-M, \quad \gamma\left(A_{+}\right)=-A_{+} e^{\vartheta M}, \quad \gamma\left(A_{-}\right)=-A_{-} e^{-\vartheta M} \\
& \gamma(N)=-N-\left(\beta_{+} / \vartheta\right) A_{+}\left(1-e^{\vartheta M}\right)-\left(\beta_{-} / \vartheta\right) A_{-}\left(1-e^{-\vartheta M}\right)
\end{aligned}
$$

and commutation relations

$$
\begin{aligned}
& {\left[N, A_{+}\right]=A_{+}-\beta_{-} V(-\vartheta), \quad\left[N, A_{-}\right]=-A_{-}-\beta_{+} V(\vartheta),} \\
& {\left[A_{-}, A_{+}\right]=M, \quad[M, \cdot]=0,}
\end{aligned}
$$

where

$$
V(x):=\frac{1}{x^{2}}\left(e^{x M}-1-x M\right)
$$

Note that $\lim _{x \rightarrow 0} V(x)=M^{2} / 2$. The quantum analogue of (2.2):

$$
C_{\vartheta, \beta_{+}, \beta_{-}}=2 N M-A_{+} A_{-}-A_{-} A_{+}+2 \beta_{-} V(-\vartheta) A_{-}-2 \beta_{+} V(\vartheta) A_{+},
$$

belongs to the center of $U_{\vartheta, \beta_{+}, \beta_{-}}^{(I I n)}\left(h_{4}\right)$.

It is worth remarking that this quantum oscillator algebra can be related with the results of [12]: $U_{\vartheta, \beta_{+}, \beta_{-}}^{(I I n)}\left(h_{4}\right)$ can be seen as a Type II case with $p \equiv \vartheta, q \equiv-\vartheta$, $b \equiv \beta_{-}$and $c \equiv-\beta_{+}$. Moreover,

Proposition 5. The element

$$
\begin{aligned}
& R=\exp \{r\}=\exp \left\{\vartheta N \wedge M+\beta_{+} A_{+} \wedge M+\beta_{-} A_{-} \wedge M\right\} \\
& =\exp \left\{-M \otimes\left(\vartheta N+\beta_{+} A_{+}+\beta_{-} A_{-}\right)\right\} \exp \left\{\left(\vartheta N+\beta_{+} A_{+}+\beta_{-} A_{-}\right) \otimes M\right\}
\end{aligned}
$$


satisfies both the quantum YBE and relation (4.24), so it is a universal $R$-matrix for $U_{\vartheta, \beta_{+}, \beta_{-}}^{(I I n)}\left(h_{4}\right)$.

Since $M$ is a central generator, it is clear that $(4.36)$ is a solution of the quantum YBE. The proof for property (4.24) is sketched in Appendix A. In the matrix representation (2.3) we get:

$$
D(R)=I \otimes I+\vartheta D(N) \wedge D(M)+\beta_{+} D\left(A_{+}\right) \wedge D(M)+\beta_{-} D\left(A_{-}\right) \wedge D(M) .
$$

The FRT prescription leads now to another multiparametric quantum deformation of the algebra of the smooth functions on the oscillator group $F u n_{\vartheta, \beta_{+}, \beta_{-}}^{(I I n)}\left(H_{4}\right)$, given by coproduct (4.27), counit (4.28), antipode (4.29) and the non vanishing commutation rules

$$
\left[\hat{a}_{+}, \hat{m}\right]=-\vartheta \hat{a}_{+}+\beta_{+}\left(e^{\hat{n}}-1\right), \quad\left[\hat{a}_{-}, \hat{m}\right]=\vartheta \hat{a}_{-}+\beta_{-}\left(e^{-\hat{n}}-1\right) .
$$

The classical limit (in the three parameters) is Fun $\left(H_{4}\right)$ and, once more, commutators (4.38) are a Weyl quantization of the Poisson-Lie brackets written in table II.

\subsection{Standard type II: $U_{z}^{(s)}\left(h_{4}\right)$}

The classical $r$-matrix which solves the classical YBE and underlies the quantum oscillator algebra obtained in [8, 11] by a contraction method can be expressed in our notation as:

$$
r=-z(N \otimes M+M \otimes N)+2 z A_{-} \otimes A_{+} .
$$

Its symmetric $\left(r_{+}\right)$and skew-symmetric $\left(r_{-}\right)$parts are:

$$
\begin{aligned}
& r_{+}=(r+\sigma \circ r) / 2=z\left(A_{-} \otimes A_{+}+A_{+} \otimes A_{-}\right)-z(N \otimes M+M \otimes N), \\
& r_{-}=(r-\sigma \circ r) / 2=z A_{-} \wedge A_{+} .
\end{aligned}
$$

The symmetric part $r_{+}$corresponds to the element $\eta$ (3.12) with the parameters $\tau_{1}=-z$ and $\tau_{2}=0$. On the other hand, $r_{-}$can be identified with a standard classical $r$-matrix of type II with parameters $\vartheta=\beta_{+}=\beta_{-}=0$ and $\xi \equiv-z$ (see table I). Both the standard $r$-matrix (which coincides with $r_{-}(4.41)$ ) and the non antisymmetric one (4.39) give rise to the same oscillator bialgebra with cocommutators:

$$
\delta(N)=\delta(M)=0, \quad \delta\left(A_{+}\right)=z A_{+} \wedge M, \quad \delta\left(A_{-}\right)=z A_{-} \wedge M
$$

The associated non vanishing Poisson-Lie brackets (see table II) are:

$$
\left\{a_{+}, m\right\}=z a_{+}, \quad\left\{a_{-}, m\right\}=z a_{-} .
$$

The quantum deformation of this coboundary oscillator bialgebra is given by: 
Proposition 6. The quantum algebra which quantizes the standard bialgebra generated by (4.39) has a Hopf structure denoted by $U_{z}^{(s)}\left(h_{4}\right)$ and characterized by the coproduct, counit, antipode

$$
\begin{gathered}
\Delta(N)=1 \otimes N+N \otimes 1, \quad \Delta\left(A_{+}^{\prime}\right)=e^{-z M} \otimes A_{+}^{\prime}+A_{+}^{\prime} \otimes 1, \\
\Delta(M)=1 \otimes M+M \otimes 1, \quad \Delta\left(A_{-}\right)=1 \otimes A_{-}+A_{-} \otimes e^{z M} ; \\
\epsilon(X)=0, \quad X \in\left\{N, A_{+}^{\prime}, A_{-}, M\right\} ; \\
\gamma(N)=-N, \quad \gamma(M)=-M, \quad \gamma\left(A_{+}^{\prime}\right)=-A_{+}^{\prime} e^{z M}, \quad \gamma\left(A_{-}\right)=-A_{-} e^{-z M} ;
\end{gathered}
$$

together with the commutation relations

$$
\left[N, A_{+}^{\prime}\right]=A_{+}^{\prime}, \quad\left[N, A_{-}\right]=-A_{-}, \quad\left[A_{-}, A_{+}^{\prime}\right]=\frac{\sinh (z M)}{z}, \quad[M, \cdot]=0 .
$$

The quantum Casimir is:

$$
C_{z}=2 N \frac{\sinh (z M)}{z}-A_{+}^{\prime} A_{-}-A_{-} A_{+}^{\prime}
$$

The coproducts (4.44) are just those given in table III but written in terms of a new generator $A_{+}^{\prime}=e^{\xi M} A_{+}$where $\xi=-z$. In this case the universal $R$-matrix adopts a much simpler form than the one already known from [8, 11. Namely,

$$
\begin{aligned}
R= & \exp \{-z(N \otimes M+M \otimes N)\} \exp \left\{2 z A_{-} \otimes A_{+}^{\prime}\right\} \\
& =\exp \{-z N \otimes M\} \exp \{-z M \otimes N\} \exp \left\{2 z A_{-} \otimes A_{+}^{\prime}\right\} .
\end{aligned}
$$

It is worth remarking that all the quantum $R$-matrices given in this section are obtained via a straightforward exponentiation process from their classical counterparts (compare, for instance, (4.49) to (4.39)).

The FRT prescription can be applied leading to the commutation rules of the quantum group $\operatorname{Fun}_{z}^{(s)}\left(H_{4}\right)$ by taking into account that (4.49) in the matrix representation (2.3) is just

$$
D(R)=I \otimes I+2 z D\left(A_{-}\right) \otimes D\left(A_{+}^{\prime}\right)-z(D(N) \otimes D(M)+D(M) \otimes D(N)) ;
$$

(note that $D\left(A_{+}^{\prime}\right) \equiv D(N)$ ). In this way, the non vanishing commutators of $\operatorname{Fun}_{z}^{(s)}\left(H_{4}\right)$ read:

$$
\left[\hat{a}_{+}, \hat{m}\right]=z \hat{a}_{+}, \quad\left[\hat{a}_{-}, \hat{m}\right]=z \hat{a}_{-},
$$

and correspond to a Weyl quantization of the Poisson-Lie brackets (4.43).

\section{Concluding remarks}

We have presented a systematic procedure in order to study the coboundary Lie bialgebras of the oscillator algebra. The first order deformations given by the corresponding cocomutators have been used to construct, by a sort of "exponentiation" 
process, multiparametric quantum deformations of the oscillator algebra. We point out that we have not treated the question of the equivalence of the coboundary oscillator bialgebras we have obtained, indeed this is actually a problem by itself. For instance, from an algebraic point of view, bialgebras of types $\mathrm{I}_{+}$and $\mathrm{I}_{-}$can be related by interchanging generators $A_{+}$and $A_{-}$, although this result is not so straightforward if we look at their corresponding Poisson-Lie groups.

It is worth stressing that, in the case here analysed, the complete (and rich) classification of the classical $r$-matrices (and, therefore, of the corresponding Poisson structures on the oscillator group) is easily obtained. This seems to indicate that, at least for Lie algebras with a low enough dimension, the complete solution of the modified classical YBE for an arbitrary skew element of $g \otimes g$ can be explicitly deduced giving rise to a great amount of new results.

This kind of procedure is complementary (and dual) to that developed in 12, since it allows us to focus on the deformation at the quantum algebra level and looking for universal quantum $R$-matrices. In fact, given a skew solution $r$ of the modified classical YBE and a matrix representation $D$ of the quantum algebra, the element $D(R)=1+z D(r)$ will lead us to the corresponding $R$-matrix method.

This approach can be seen as a part of a research program that, in order to construct and study quantum algebras, tries to extract as much information as possible from the associated Lie bialgebras (as far as contraction methods are concerned, see for instance [19]). It would be interesting to apply it to other physically interesting algebras whose coboundary bialgebra structures are not well known, among them, we would like to mention the Schrödinger, optical and Galilean algebras, also with the aim of obtaining some (universal) quantum deformations.

\section{Acknowledgements}

The authors acknowledge M. Santander and M.A. del Olmo a careful reading of the manuscript and many helpful suggestions as well as the referees for some

pertinent comments. This work has been partially supported by DGICYT (Projects PB92-0255 and PB94-1115) from the Ministerio de Educación y Ciencia de España.

\section{Appendix A}

The main steps necessary to prove that the $R$-matrix (4.36) verifies the property (4.24) for the generators $A_{+}, A_{-}$and $N$ (for $M$ the proof is trivial) are as follows. We perform the computations by writing the $R$-matrix in terms of two exponentials $R=\exp \{-M \otimes W\} \exp \{W \otimes M\}$, where $W \equiv \vartheta N+\beta_{+} A_{+}+\beta_{-} A_{-}$. We note that

$$
\begin{aligned}
& \exp \{W \otimes M\} \Delta\left(A_{+}\right) \exp \{-W \otimes M\} \\
& =1 \otimes A_{+}+A_{+} \otimes 1-\beta_{-} V(-\vartheta) \otimes\left(1-e^{-\vartheta M}\right)+\left(\beta_{-} / \vartheta\right) M \otimes\left(1-e^{-\vartheta M}\right) \\
& =\Delta_{0}\left(A_{+}\right)+\left(\beta_{-} / \vartheta^{2}\right)\left(1-e^{-\vartheta M}\right) \otimes\left(1-e^{-\vartheta M}\right) .
\end{aligned}
$$


Since the second term of (A.1) is central, we compute:

$$
\begin{aligned}
& \exp \{-M \otimes W\} \Delta_{0}\left(A_{+}\right) \exp \{M \otimes W\} \\
& =e^{-\vartheta M} \otimes A_{+}+A_{+} \otimes 1+\beta_{-}\left(1-e^{-\vartheta M}\right) \otimes V(-\vartheta)-\left(\beta_{-} / \vartheta\right)\left(1-e^{-\vartheta M}\right) \otimes M \\
& =\sigma \circ \Delta\left(A_{+}\right)-\left(\beta_{-} / \vartheta^{2}\right)\left(1-e^{-\vartheta M}\right) \otimes\left(1-e^{-\vartheta M}\right) .
\end{aligned}
$$

From these expressions $R \Delta\left(A_{+}\right) R^{-1}=\sigma \circ \Delta\left(A_{+}\right)$is easily derived. The proof for $A_{-}$is rather similar, and for the generator $N$ we shall have:

$$
\begin{aligned}
& \exp \{W \otimes M\} \Delta(N) \exp \{-W \otimes M\} \\
& =1 \otimes N+N \otimes 1-\left(\beta_{+} \beta_{-} / \vartheta^{2}\right)\left\{\vartheta V(\vartheta) \otimes\left(1-e^{\vartheta M}\right)+M \otimes\left(1-e^{-\vartheta M}\right)\right\} \\
& \quad+\left(\beta_{+} \beta_{-} / \vartheta^{2}\right)\left\{\vartheta V(-\vartheta) \otimes\left(1-e^{\vartheta M}\right)-M \otimes\left(1-e^{-\vartheta M}\right)\right\} \\
& =\Delta_{0}(N)+\left(\beta_{+} \beta_{-} / \vartheta^{3}\right)\left\{\left(1-e^{\vartheta M}\right) \otimes\left(1-e^{\vartheta M}\right)-\left(1-e^{-\vartheta M}\right) \otimes\left(1-e^{-\vartheta M}\right)\right\} .
\end{aligned}
$$

Now we compute

$$
\begin{aligned}
& \exp \{-M \otimes W\} \Delta_{0}(N) \exp \{M \otimes W\} \\
&=\Delta_{0}(N)+\left(\beta_{+} / \vartheta\right)\left(1-e^{-\vartheta M}\right) \otimes A_{+}+\left(\beta_{-} / \vartheta\right)\left(1-e^{\vartheta M}\right) \otimes A_{-} \\
&+\left(\beta_{+} \beta_{-} / \vartheta\right)\left\{\left(1-e^{\vartheta M}\right) \otimes V(\vartheta)-\left(1-e^{-\vartheta M}\right) \otimes V(-\vartheta)\right\} \\
&+\left(\beta_{+} \beta_{-} / \vartheta^{2}\right)\left(2-e^{\vartheta M}-e^{-\vartheta M}\right) \otimes M \\
&= \sigma \circ \Delta(N)-\left(\beta_{+} \beta_{-} / \vartheta^{3}\right)\left\{\left(1-e^{\vartheta M}\right) \otimes\left(1-e^{\vartheta M}\right)-\left(1-e^{-\vartheta M}\right) \otimes\left(1-e^{-\vartheta M}\right)\right\},
\end{aligned}
$$

to obtain again $R \Delta(N) R^{-1}=\sigma \circ \Delta(N)$. 
Table I. Coboundary oscillator Lie bialgebras.

\begin{tabular}{|c|c|c|}
\hline & \multicolumn{2}{|c|}{ Type $\mathbf{I}_{+}$} \\
\hline & $\begin{array}{c}\text { Standard } \\
\left(\alpha_{+} \neq 0 \text { and } \alpha_{+} \beta_{-}-\vartheta^{2} \neq 0\right)\end{array}$ & $\begin{array}{l}\text { Non-standard } \\
\quad\left(\alpha_{+} \neq 0\right)\end{array}$ \\
\hline$r$ & $\begin{array}{c}\alpha_{+} N \wedge A_{+}+\vartheta\left(N \wedge M-A_{+} \wedge A_{-}\right) \\
+\beta_{+} A_{+} \wedge M+\beta_{-} A_{-} \wedge M\end{array}$ & $\begin{array}{l}\alpha_{+} N \wedge A_{+}+\vartheta\left(N \wedge M-A_{+} \wedge A_{-}\right) \\
\quad+\beta_{+} A_{+} \wedge M+\left(\vartheta^{2} / \alpha_{+}\right) A_{-} \wedge M\end{array}$ \\
\hline \multirow[t]{3}{*}{$\begin{array}{c}\delta(N) \\
\delta\left(A_{+}\right) \\
\delta\left(A_{-}\right) \\
\delta(M) \\
\end{array}$} & $\begin{array}{l}\alpha_{+} N \wedge A_{+}-\beta_{-} A_{-} \wedge M+\beta_{+} A_{+} \wedge M \\
0 \\
\alpha_{+}\left(A_{-} \wedge A_{+}+N \wedge M\right)+2 \vartheta A_{-} \wedge M \\
0\end{array}$ & $\begin{array}{l}\alpha_{+} N \wedge A_{+}-\left(\vartheta^{2} / \alpha_{+}\right) A_{-} \wedge M+\beta_{+} A_{+} \wedge M \\
\quad 0 \\
\alpha_{+}\left(A_{-} \wedge A_{+}+N \wedge M\right)+2 \vartheta A_{-} \wedge M \\
\quad 0\end{array}$ \\
\hline & \multicolumn{2}{|c|}{ Type $I_{-}$} \\
\hline & $\begin{array}{c}\text { Standard } \\
\left(\alpha_{-} \neq 0 \text { and } \alpha_{-} \beta_{+}-\vartheta^{2} \neq 0\right)\end{array}$ & $\begin{array}{l}\text { Non-standard } \\
\quad\left(\alpha_{-} \neq 0\right)\end{array}$ \\
\hline$r$ & $\begin{array}{c}\alpha_{-} N \wedge A_{-}+\vartheta\left(N \wedge M+A_{+} \wedge A_{-}\right) \\
+\beta_{+} A_{+} \wedge M+\beta_{-} A_{-} \wedge M\end{array}$ & $\begin{aligned} \alpha_{-} & N \wedge A_{-}+\vartheta\left(N \wedge M+A_{+} \wedge A_{-}\right) \\
& +\left(\vartheta^{2} / \alpha_{-}\right) A_{+} \wedge M+\beta_{-} A_{-} \wedge M\end{aligned}$ \\
\hline $\begin{array}{c}\delta(N) \\
\delta\left(A_{+}\right) \\
\delta\left(A_{-}\right) \\
\delta(M)\end{array}$ & $\begin{array}{l}-\alpha_{-} N \wedge A_{-}+\beta_{+} A_{+} \wedge M-\beta_{-} A_{-} \wedge M \\
-\alpha_{-}\left(A_{+} \wedge A_{-}+N \wedge M\right)-2 \vartheta A_{+} \wedge M \\
\quad 0 \\
\quad 0\end{array}$ & $\begin{array}{l}-\alpha_{-} N \wedge A_{-}+\left(\vartheta^{2} / \alpha_{-}\right) A_{+} \wedge M-\beta_{-} A_{-} \wedge M \\
-\alpha_{-}\left(A_{+} \wedge A_{-}+N \wedge M\right)-2 \vartheta A_{+} \wedge M \\
\quad 0 \\
\quad 0\end{array}$ \\
\hline \multirow{2}{*}{$\delta(M)$} & \multicolumn{2}{|c|}{ Type II } \\
\hline & $\begin{array}{c}\text { Standard } \\
(\xi \neq 0)\end{array}$ & Non-standard \\
\hline$r$ & $\begin{aligned} \vartheta N & \wedge M+\xi A_{+} \wedge A_{-} \\
& +\beta_{+} A_{+} \wedge M+\beta_{-} A_{-} \wedge M\end{aligned}$ & $\vartheta N \wedge M+\beta_{+} A_{+} \wedge M+\beta_{-} A_{-} \wedge M$ \\
\hline $\begin{array}{c}\delta(N) \\
\delta\left(A_{+}\right) \\
\delta\left(A_{-}\right) \\
\delta(M)\end{array}$ & $\begin{array}{l}\beta_{+} A_{+} \wedge M-\beta_{-} A_{-} \wedge M \\
-(\vartheta+\xi) A_{+} \wedge M \\
(\vartheta-\xi) A_{-} \wedge M \\
\quad 0\end{array}$ & $\begin{array}{l}\beta_{+} A_{+} \wedge M-\beta_{-} A_{-} \wedge M \\
-\vartheta A_{+} \wedge M \\
\vartheta A_{-} \wedge M \\
\quad 0\end{array}$ \\
\hline
\end{tabular}


Table II. Poisson-Lie brackets on the oscillator group.

\begin{tabular}{|c|c|c|}
\hline & \multicolumn{2}{|c|}{ Type $\mathbf{I}_{+}$} \\
\hline & $\begin{array}{c}\text { Standard } \\
\left(\alpha_{+} \neq 0 \text { and } \alpha_{+} \beta_{-}-\vartheta^{2} \neq 0\right)\end{array}$ & $\begin{array}{l}\text { Non-standard } \\
\quad\left(\alpha_{+} \neq 0\right)\end{array}$ \\
\hline \multirow{8}{*}{$\begin{array}{c}\left\{n, a_{+}\right\} \\
\left\{n, a_{-}\right\} \\
\left\{a_{-}, a_{+}\right\} \\
\{n, m\} \\
\left\{a_{+}, m\right\} \\
\left\{a_{-}, m\right\} \\
\end{array}$} & $\alpha_{+}\left(e^{n}-1\right)$ & $\alpha_{+}\left(e^{n}-1\right)$ \\
\hline & 0 & 0 \\
\hline & $\alpha_{+} a_{-}$ & $\alpha_{+} a_{-}$ \\
\hline & $\alpha_{+} a_{-}$ & $\alpha_{+} a_{-}$ \\
\hline & $\alpha_{+} a_{-} a_{+}+\beta_{+}\left(e^{n}-1\right)$ & $\alpha_{+} a_{-} a_{+}+\beta_{+}\left(e^{n}-1\right)$ \\
\hline & $-\alpha_{+} a_{-}^{+}+2 v a_{-}+\beta_{-}\left(e^{-}-1\right)$ & $-\alpha_{+} a_{-}^{-}+2 \vartheta a_{-}+\left(\vartheta^{2} / \alpha_{+}\right)\left(e^{n}-1\right)$ \\
\hline & \multicolumn{2}{|c|}{ Type $I_{-}$} \\
\hline & $\begin{array}{c}\text { Standard } \\
\left(\alpha_{-} \neq 0 \text { and } \alpha_{-} \beta_{+}-\vartheta^{2} \neq 0\right)\end{array}$ & $\begin{array}{l}\text { Non-standard } \\
\quad\left(\alpha_{-} \neq 0\right)\end{array}$ \\
\hline \multirow{8}{*}{$\begin{array}{c}\left\{n, a_{+}\right\} \\
\left\{n, a_{-}\right\} \\
\left\{a_{-}, a_{+}\right\} \\
\{n, m\} \\
\left\{a_{+}, m\right\} \\
\left\{a_{-}, m\right\} \\
\end{array}$} & 0 & 0 \\
\hline & $\alpha_{-}\left(e^{-n}-1\right)$ & $\alpha_{-}\left(e^{-n}-1\right)$ \\
\hline & $\alpha_{-} a_{+}$ & $\alpha_{-} a_{+}$ \\
\hline & $-\alpha_{-} a_{+} e^{-n}$ & $-\alpha_{-} a_{+} e^{-n}$ \\
\hline & $-2 \vartheta a_{+}+\beta_{+}\left(e^{n}-1\right)$ & $-2 \vartheta a_{+}+\left(\vartheta^{2} / \alpha_{-}\right)\left(e^{n}-1\right)$ \\
\hline & $\beta_{-}\left(e^{-n}-1\right)$ & $\beta_{-}\left(e^{-n}-1\right)$ \\
\hline & \multicolumn{2}{|c|}{ Type II } \\
\hline & $\begin{array}{l}\text { Standard } \\
(\xi \neq 0)\end{array}$ & Non-standard \\
\hline$\left\{n, a_{+}\right\}$ & 0 & $\overline{0}$ \\
\hline$\left\{n, a_{-}\right\}$ & 0 & 0 \\
\hline$\left\{a_{-}, a_{+}\right\}$ & 0 & 0 \\
\hline$\{n, m\}$ & 0 & 0 \\
\hline$\left\{a_{+}, m\right\}$ & $-(\vartheta+\xi) a_{+}+\beta_{+}\left(e^{n}-1\right)$ & $-\vartheta a_{+}+\beta_{+}\left(e^{n}-1\right)$ \\
\hline$\left\{a_{-}, m\right\}$ & $(\vartheta-\xi) a_{-}+\beta_{-}\left(e^{-n}-1\right)$ & $\vartheta a_{-}+\beta_{-}\left(e^{-n}-1\right)$ \\
\hline
\end{tabular}


Table III. Coproducts for QUEA of the oscillator algebra.

\begin{tabular}{|c|}
\hline Type $\mathbf{I}_{+}$ \\
\hline$\left(\alpha_{+} \neq 0\right.$ and $\left.\alpha_{+} \beta_{-}-\vartheta^{2} \neq 0\right)$ \\
\hline $\begin{array}{l}\Delta\left(A_{+}\right)=1 \otimes A_{+}+A_{+} \otimes 1 \\
\Delta\left(\begin{array}{c}N^{\prime} \\
A_{-}\end{array}\right)=\left(\begin{array}{c}1 \otimes N^{\prime} \\
1 \otimes A_{-}\end{array}\right)+\sigma\left(\exp \left\{\left(\begin{array}{cc}\alpha_{+} A_{+} & -\beta_{-} M \\
\alpha_{+} M & \alpha_{+} A_{+}+2 \vartheta M\end{array}\right)\right\} \dot{\otimes}\left(\begin{array}{c}N^{\prime} \\
A_{-}\end{array}\right)\right) \\
N^{\prime}=N-\left(\beta_{+} / \alpha_{+}\right) M\end{array}$ \\
\hline Non-standard: $\quad U_{\alpha_{+}, \vartheta, \beta_{+}}^{\left(I_{+} n\right)}\left(h_{4}\right)$ \\
\hline $\begin{array}{l}\Delta\left(A_{+}\right)=1 \otimes A_{+}+A_{+} \otimes 1 \quad \Delta(M)=1 \otimes M+M \otimes 1 \\
\Delta(N)=1 \otimes N+N \otimes(1-\vartheta M) \exp \left\{\alpha_{+} A_{+}+\vartheta M\right\}-\left(\vartheta^{2} / \alpha_{+}\right) A_{-} \otimes M \exp \left\{\alpha_{+} A_{+}+\vartheta M\right\} \\
\quad+\left(\beta_{+} / \alpha_{+}\right) M \otimes\left(1-(1-\vartheta M) \exp \left\{\alpha_{+} A_{+}+\vartheta M\right\}\right) \\
\begin{aligned} \Delta\left(A_{-}\right)= & 1 \otimes A_{-}+A_{-} \otimes(1+\vartheta M) \exp \left\{\alpha_{+} A_{+}+\vartheta M\right\} \\
& +\left(\alpha_{+} N-\beta_{+} M\right) \otimes M \exp \left\{\alpha_{+} A_{+}+\vartheta M\right\}\end{aligned}\end{array}$ \\
\hline Type $I_{-}$ \\
\hline$\left(\alpha_{-} \neq 0\right.$ and $\left.\alpha_{-} \beta_{+}-\vartheta^{2} \neq 0\right)$ \\
\hline $\begin{array}{l}\Delta\left(A_{-}\right)=1 \otimes A_{-}+A_{-} \otimes 1 \\
\Delta\left(\begin{array}{c}N^{\prime} \\
A_{+}\end{array}\right)=\left(\begin{array}{c}1 \otimes N^{\prime} \\
1 \otimes A_{+}\end{array}\right)+\sigma\left(\exp \left\{\left(\begin{array}{cc}-\alpha_{-} A_{-} & \beta_{+} M \\
-\alpha_{-} M & -\alpha_{-} A_{-}-2 \vartheta M\end{array}\right)\right\} \dot{\otimes}\left(\begin{array}{c}N^{\prime} \\
A_{+}\end{array}\right)\right) \\
N^{\prime}=N-\left(\beta_{-} / \alpha_{-}\right) M\end{array}$ \\
\hline Non-standard: $\quad U_{\alpha_{-}, \beta_{3} \beta_{-}}^{\left(I_{-} n\right)}\left(h_{4}\right)$ \\
\hline $\begin{array}{l}\Delta\left(A_{-}\right)=1 \otimes A_{-}+A_{-} \otimes 1 \quad \Delta(M)=1 \otimes M+M \otimes 1 \\
\begin{aligned} \Delta(N)= & 1 \otimes N+N \otimes(1+\vartheta M) \exp \left\{-\alpha_{-} A_{-}-\vartheta M\right\}+\left(\vartheta^{2} / \alpha_{-}\right) A_{+} \otimes M \exp \left\{-\alpha_{-} A_{-}-\vartheta M\right\} \\
& +\left(\beta_{-} / \alpha_{-}\right) M \otimes\left(1-(1+\vartheta M) \exp \left\{-\alpha_{-} A_{-}-\vartheta M\right\}\right) \\
\Delta\left(A_{+}\right)= & 1 \otimes A_{+}+A_{+} \otimes(1-\vartheta M) \exp \left\{-\alpha_{-} A_{-}-\vartheta M\right\} \\
& \quad\left(\alpha_{-} N-\beta_{+} M\right) \otimes M \exp \left\{-\alpha_{-} A_{-}-\vartheta M\right\}\end{aligned}\end{array}$ \\
\hline Type II \\
\hline Standard: $\quad U_{\vartheta, \xi, \beta_{+}, \beta_{-}}^{(I I s)}\left(h_{4}\right)$ \\
\hline $\begin{array}{l}\Delta(M)=1 \otimes M+M \otimes 1 \\
\Delta\left(A_{+}\right)=1 \otimes A_{+}+A_{+} \otimes \exp \{-(\vartheta+\xi) M\} \\
\Delta\left(A_{-}\right)=1 \otimes A_{-}+A_{-} \otimes \exp \{(\vartheta-\xi) M\} \\
\begin{aligned} \Delta(N)=1 \otimes N+N \otimes 1+\left(\beta_{+} /(\vartheta+\xi)\right) A_{+} \otimes(1-\exp \{-(\vartheta+\xi) M\}) \\
\quad+\left(\beta_{-} /(\vartheta-\xi)\right) A_{-} \otimes(1-\exp \{(\vartheta-\xi) M\})\end{aligned}\end{array}$ \\
\hline Non-standard: $\quad U_{\vartheta, \beta_{+}, \beta_{-}}^{(I I n)}\left(h_{4}\right)$ \\
\hline $\begin{array}{l}\Delta(M)=1 \otimes M+M \otimes 1 \\
\Delta\left(A_{+}\right)=1 \otimes A_{+}+A_{+} \otimes \exp \{-\vartheta M\} \\
\Delta\left(A_{-}\right)=1 \otimes A_{-}+A_{-} \otimes \exp \{\vartheta M\} \\
\Delta(N)=1 \otimes N+N \otimes 1+\left(\beta_{+} / \vartheta\right) A_{+} \otimes(1-\exp \{-\vartheta M\})+\left(\beta_{-} / \vartheta\right) A_{-}\end{array}$ \\
\hline
\end{tabular}




\section{References}

[1] Greenberg O W 1991 Phys. Rev. D 434111

[2] Avancini S S and Krein G 1995 J. Phys. A: Math. Gen. 28685

[3] Spiridonov V 1992 Phys. Rev. Lett. 69398

[4] Bonatsos D, Daskaloyannis and Kokkotas K 1993 Phys. Rev. A 48 R3407

[5] Bonatsos D, Daskaloyannis and Kokkotas K 1994 Phys. Rev. A 503700

[6] Floreanini R, Le Tourneaux J and Vinet L 1995 J. Phys. A: Math. Gen. 28 L287

[7] Celeghini E, Rasetti M and Vitiello G 1995 J. Phys. A: Math. Gen. 28 L239

[8] Gómez C and Sierra G 1993 J. Math. Phys. 342119

[9] Lyakhovsky V and Mudrov A 1992 J. Phys. A: Math. Gen. 25 L1139

[10] Ballesteros A, Herranz F J, Pereña C M, del Olmo M A and Santander M 1995 J. Phys. A: Math. Gen. 287113

[11] Celeghini E, Giachetti R, Sorace E and Tarlini M 1991 J. Math. Phys. 321155

[12] Hussin V, Lauzon A and Rideau G Oscillator quantum groups from R-matrix method J. Phys. A: Math. Gen. to appear

[13] Reshetikhin N Y, Takhtadzhyan L A and Faddeev L D 1990 Leningrad Math. J. 1193

[14] Ballesteros A, Celeghini E, Giachetti R, Sorace E and Tarlini M 1993 J. Phys. A: Math. Gen. 267495

[15] Hussin V, Lauzon A and Rideau G 1994 Lett. Math. Phys. 31159

[16] Tjin T 1992 Int. J. Mod. Phys. A 76175

[17] Ohn C 1992 Lett. Math. Phys. 2585

[18] Ballesteros A, Herranz F J, del Olmo M A, and Santander M 1995 J. Phys. A: Math. Gen. 28941

[19] Ballesteros A, Gromov N A, Herranz F J, del Olmo M A, and Santander M 1995 J. Math. Phys. 365916 